\title{
Evaluation of Hexane Extract of Tuber of Root of Cyperus rotundus Linn (Cyperaceae) for Repellency against Mosquito Vectors
}

\author{
S. P. Singh, K. Raghavendra, and A. P. Dash \\ National Institute of Malaria Research (Indian Council of Medical Research), 22-Shamnath Marg, New Delhi 110054, India \\ Correspondence should be addressed to S. P. Singh, sspmrc@rediffmail.com
}

Received 12 March 2009; Revised 25 June 2009; Accepted 27 August 2009

Recommended by Wej Choochote

Hexane extract of tuber of plant Cyperus rotundus (Cyperaceae) was screened under laboratory conditions for repellent activity against mosquito vector Anopheles culicifacies Giles species A (Diptera: Culicidae), Anopheles stephensi Liston (Diptera: Culicidae), and Culex quinquefasciatus Say (Diptera: Culicidae). The Cyperus rotundus tuber extract was used to determine their effect on mosquito vector, and comparison with the DEET (NN Diethyl 1-3 methyl Benzamide, formerly known as diethyl 1-m-toluamide). The tuber extracts showed more effective at all the dose. Result obtained from the laboratory experiment showed that the tuber extracts are more effective for repellency of allthe mosquito vector even at low dose. Clear dose response relationships were established with the highest dose of $10 \%$ tuber extract evoking $100 \%$ repellency. Percent protection obtained against An. culicifacies Giles species A 100\% repellency in 4 hours, 6 hours, An. stephensi 100\% repellency in 6 hours and Cx. quinquefasciatus was 100\% repellency in 6 hours at the 10\% concentration. Against DEET- 2.5\% An. culicifacies A 100\% repellency in 1 hour, 2 hours, 6 hours, An. stephensi have shown 100\% repellency in 6 hours, and Culex quinquefasciatus have shown 100\% repellency in 1 hour, 2 hours, 6 hours. The consolidated data of the repellency observed in different species is given and it is evident that the over all repellency rates varied between 80 and $100 \%$ for different repellents concentrations (2.5\%, 5\%, and 10\%). The extract can be applied as an effective personal protective measure against mosquito bites.

Copyright ( 2009 S. P. Singh et al. This is an open access article distributed under the Creative Commons Attribution License, which permits unrestricted use, distribution, and reproduction in any medium, provided the original work is properly cited.

\section{Introduction}

Malaria contributes the major disease burden and its control is hampered many operational and technical reasons and among the technical reason insecticidal resistance, namely, development of insecticide resistance in malaria vectors to the commonly used synthetic chemical insecticides in public health sprays has made the disease control more difficult [1]. Mosquito population has not been reduced significantly after continuous use of synthetic insecticides and Anophelines retain their potential to support the outbreak and development of malaria in endemic areas. Anopheles stephensi Liston and Anopheles culicifacies Giles are two major vectors of malaria in India and later is a sibling species complex. There are five sibling species present named A, B, C, D, and E, of which species $A, C, D$, and $E$ are vectors while $B$ is a poor vector [2]. Culex quinquefasciatus Say transmits Filariasis while dengue fever and DHF are transmitted by the vector Aedes aegypti Linn.

In contrast to vaccines and chemoprophylaxis as means of personal protection, repellents are convenient, inexpensive, and afford advantages in protection against a wide range of vector [3]. They are also the primary means of mosquito-borne disease prevention available in areas where vector control is not practical $[4,5]$. The majority of commercial repellent products contain the DEET (Diethyl 1-3 methyl Benzamide, formerly known as diethyl 1$\mathrm{m}$ toluamide), which was first Synthesized in 1954 [6]. Although effective, Deet is not the ideal product, as allergic and toxic effects have been documented [4, 7] and its solvent characteristics can damage plastic and other synthetic materials. Deet, in combination with certain other agents, is suspected of causing Gulf War syndrome [8,9], although this matter is still controversial. Because of the undesirable 
effect of Deet, research was actively carried out to find an alternative compound that is safer to use and equally or more effective (Robert et al. [10]), [11-13]. One promising new repellent is the piperidine compound, A13-37220, which provides equal or better protection against certain mosquitoes than that obtained with Deet [13-15]. Despite the significant repellency of A13-37220, there are concerns over its safety.

The repellent potential of plant to mosquitoes and other pest insects has been well known both prior to [16] and after [17] the advent of synthetic chemicals, Various botanical substances, Cymbopogan spp. [18, 19], Eucalyptus maculate citriodon [20], Azadirachta indica [21], Pelargonium citrosum [22], Lantana camara [12], and Mentha piperita [23] have been reported as being repellent against adult mosquitoes. Some promising essential oils such as citronella. Lemon eucalyptus, neem, and peppermint oils are derived from these plants and they are currently available in several commercially formulated repellents. However, their repellency is still lower in both efficacy and duration than that in currently used chemicals such as Deet and A13-37220. Nevertheless, the possible health risks associated with use of these chemicals should be taken in to consideration. With this in mind, the present study was carried out to investigate the repellent activity of the Indian medicinal plant, Cyperus rotundus against the mosquito Anopheles culicifacies Giles species A (Diptera: Culicidae), Anopheles stephensi Liston (Diptera: Culicidae), and Culex quinquefasciatus Say (Diptera: Culicidae). This may provide a new and promising repellent that can be used in the development of an economical and practical means to protect humans from the mosquito bites. Several frequently used herbal drugs are derived from the Cyperus rotundus species of the family Cyperaceae. The tuber of the root of Cyperus is commonly used as traditional drugs. Cyperus rotundus has been traditionally used in India for food and medicinal activity such as antibacterial, analgesic, antipyretic, and antiplasmodic, antibacterial, analgesic, antipyretic, antiplasmodic (Glossary of Indian medicinal plant PID-Chopra et al. [24]) Presence of highly significant anti-inflammatory and antipyretic in Cyperus rotundus suggests a very useful remedy for arthritic condition. The presence of tranquilizing, hypotensive, smooth muscle relaxant, antiemetic, anthistaminic anti-inflammatory and antipyretic activities show that the extract possesses significant pharmacological activity [25].

\section{Material and Methods}

For these studies plants were collected from rural area of district Agra of Utter Pradesh and in Delhi state of north India. The plant was identified by the National Institute of Science Communication, The Wealth of India Division, Council of Scientific and Industrial Research, New Delhi, India.

2.1. Hexane Extracts of Tuber of Cyperus Rotundus. Fresh tuber was collected and washed with water, dried in shade, and powdered. The tuber of the root of Cyoerus rotundus powdered material of this plant $(1 \mathrm{~kg})$ was subjected to extract three times in a soxhlet apparatus using solvent of hexane in ratio of $1: 3(\mathrm{w} / \mathrm{v})$ of $95 \%$ hexane at room temperature. The extract was made solvent free and the final residue of hexane extract of Cyperus rotundus obtained, lyophilized, and then kept at $-20^{\circ} \mathrm{C}$ until testing for adult repellent activity. In preparing test concentrations, extract was volumetrically diluted in absolute hexane and Tween 80 at an appropriate test concentration.

2.2. Mosquito Strains. An. culicifacies species A, An. stephensi, and $C x$. quinquefasciatus were used in this study. Mosquitoes were reared in a laboratory maintained at $27 \pm 2{ }^{\circ} \mathrm{C}$ temperatures and $70 \pm 5 \%$ relative humidity with a photoperiod of 12 : 12 hours (Light : Dark) with 90 minutes down and dusk simulation periods. Adult's mosquitoes were provided with $10 \%$ sucrose. The 5-8 days old females used for investigation of repellent activity.

2.3. Mosquito Cages. One hundred-5-8 days old-sugar-fed female mosquitoes were introduced in to the cloth cages. These mosquitoes were starved for about 15 hours prior to their introduction in to the cages. A minimum of three replicates were prepared for a given species of mosquito. Needed standardization was made for experiments as to the determination of the suitable age of the mosquito for experiments and method of recording the data (Protocols for Uniform Evaluation of Insecticides for use in Vector Control. National Institute of Malaria Research-2005http://www.mrcindia.org/).

2.4. Preparation of the Repellent and Control Replicates. $500 \mathrm{~mL}$ of $10 \%$ sugar solution was prepared in water. Sufficient quantity of bleached cotton was taken to be stacked in to a $460 \mathrm{~mL}$ Styrofoam glass. $450 \mathrm{~mL}$ of the above sugar solution was poured into the glass and the cotton was soaked. The cotton at the top was stretched out side in to circular foam. Remaining $40 \mathrm{~mL}$ was used to prepare repellent formulation. To $40 \mathrm{~mL}$ of the sugar solution required quantity of the tuber hexane extract concentrate was mixed to arrive at the desired concentrations, namely, $2.5 \%, 5 \%$, and $10 \%$ and was poured evenly on the sugar soaked cotton in the above Styrofoam glass. Similarly DEET $2.5 \%$ in $10 \%$ sugar soaked cotton was prepared for use as positive and only $10 \%$ sugar soaked cotton was used as negative controls, respectively. For tuber of root of Cyperus rotundus hexane extract, known quantity of residue extract was redissolved in hexane to make a $10 \%(\mathrm{w} / \mathrm{v})$ stock solution. Various test concentrations ranging between $2.5 \%$, $5 \%$, and $10 \%$ were prepared in double distilled water using freshly made stock solution. Controls were supplemented with the equal amount hexane required for the experiment without extracts. Tween-80 was used as an emulsifier at $0.05 \%$ concentration in the final test solution. 


\section{Repellency tests}

Experimental cages with the mosquitoes were placed in this room. In these cages, the Styrofoam glasses with cotton soaked with three different concentrations of tuber hexane extract of Cyperus rutundus namely 2.5\%, 5\%, and $10 \%$ sugar solution, DEET $2.5 \%$ (positive control) in 10\% sugar solution and $10 \%$ sugar solution (negative control) were placed in four different corners and one in the centre of the cage. Five-minute landing counts were made at $0,1,2,4,5$, and 6 hours. The cups were removed from the cage after the five minute observation at each interval of time. The cup was covered to avoid evaporation of the insecticide formulation and was placed in the refrigerator. For subsequent exposure the position of the cups were inter changed to different corners (Protocols for Uniform Evaluation of Insecticides for use in Vector Control. National Institute of Malaria Research-2005-http://www.mrcindia.org/).

3.1. Data Analysis. Observation is made in at least three replicates for the given species of the mosquito that landed and attempted to feed were recorded. No mosquito landing occurred in the initial 3 minutes the cups interchanged into the different corners. Observations were made at 30 minutes intervals. If more than 1 mosquito landing was recorded during an observation, the test of repellency was terminated and the period of repellent protection calculated as the time between the extract application and multiple mosquito landing. The landing rates of the mosquitoes on different concentration of the formulation of hexane extract of tuber of root of Cyperus rotundus (2.5, 5, and $10 \%$ (DEET 2.5\% and sugar $(10 \%)$ were recorded. Data was reported as mean of the observations for each of the formulation. Result was expressed as average landing counts per exposure interval and as repellency compared to control means using as follows [26]:

$$
\% \text { Repellency }=\frac{C-T}{C} \times 100,
$$

where $\mathrm{C}$ : the mean number of landing on negative control (10\% sugar solution); T: landing on the repellents (DEET and tuber of root of Cyperus rotundus).

\section{Results}

The consolidated data of the repellency observed in different species is given and it is evident that the overall repellency rates varied between $80-100 \%$ for different repellents, concentrations of repellents and species. The hexane extract of Cyperus rotundus was the candidate for subsequent field investigations repellent activity. Species and number of mosquitoes collected from the control are demonstrated in Table 1. A total of 100 adult mosquito comprising three species in two genera were rearing in laboratory NIMR. Table 1 summarizes the results of Cyperus rotundus laboratory testing, these results showed a highly significant difference between the mean landing counting on the treated and control. The hexane extract of tuber of the root seed showed strong repellent activity against adult mosquitoes. Percent protection obtained against Anopheles culicifacies Giles species A (100\% in 4 hours, 6 hours) Anopheles stephensi (100\% in 6 hours) and Culex quinquefasciatus were (100\% in 6 hours) at the $10 \%$ concentration. Against DEET2.5\% An. Culicifacies A 100\% repellency in 1 hour, 2 hours, 6 hours, An. stephensi have shown 100\% repellency in 6 hours, and Culex quinquefasciatus have shown 100\% repellency in 1 hour, 2 hours, 6 hours. However, testing hexane extract of Cyperus rotundus need to be carried out in the field.

Hexane extract of Cyperus rotundus was found effective in repelling the three mosquito disease vectors, Anopheles culicifacies species A, Anopheles stephensi (malaria vectors), and Culex quinquefasciatus (filaria vector) though with minor variations. The percent repellency at different observation periods ( 0 hour, 1 hour, 2 hours, 4 hours, and 6 hours ranged from 80 to $100 \%$ against different concentrations, repellents, and species.

Against hexane extract of tuber of root of Cyperus rotundus, Culex quinquefasciatus has shown more than $90 \%$ repellency to all the concentrations (except $2.5 \%$ in 0 hour, 1 hour, 2 hours) that is, 2.5, 5, and 10\%. Anopheles culicifacies species A and Anopheles stephensi have shown 90-100\% repellency against at 5\% and 10\% concentrations, while at $2.5 \%$ the repellency ranged between $75-90 \%$.

\section{Discussion}

In laboratory test, Cyperus rotundus hexane extract possessed significant repellent activity against the three species tested which is similar compared to that reported for currently used synthetic compounds such as DEET, A13-35765, A1337220 and CIC-4 [14, 27]. These chemical compounds provide better and longer protection against many biting insects and level $=0.37-25.37 \mu \mathrm{g} / \mathrm{cm}^{2}, 2-8$ hours). Repellent protection time in laboratory bioassays however can change depending on the biological characteristics of the mosquito test population. Differences in species and body size, sugar water availability, adult density in test cages, and mosquito age can affect test results [18, 28-30]. Tawatsin et al. [31] demonstrated under laboratory conditions that volatile oils derived from turmeric (curcuma longa), citronella grass (Cymbopogon winterianus), and hairy basil (Ocimum americanum) with the addition of $5 \%$ vanillin were effective in repelling both diurnal and nocturnal mosquitoes for up to six hours. The hexane extract of Cyperus rotundus was effective in reducing the vector species. When compared with the study of Sharma et al. [32], the protective effect against An. Culicifacies, An. stephensi and Culex quinquefasciatus, of hexane extracted of Cyperus rotundus seem to be higher than that of neem oil $(37.5 \%)$. Selection of a repellent for further development cannot be based on the results of any one test against a single insect because mosquito responses to repellents vary within and among species [18, 33]. The protection against $C x$ tritaeniorhynchus and $C x$. quinquefasciatus, the vectors of Japanese B encephalitis $[34,35]$ and filariasis $[11,36]$, respectively, is considered as satisfactory. The hexane-extracted Cyperus rotundus may protect against 
TABle 1: Percent repellency of hexane extract of tuber of root of Cyperus rotundus Linn against three important mosquito vectors.

\begin{tabular}{|c|c|c|c|c|c|c|}
\hline \multirow{2}{*}{ Species Replicate } & \multirow{2}{*}{ Doses \% } & \multicolumn{5}{|c|}{ Repellency in hours } \\
\hline & & 0 hour & 1 hour & 2 hours & 4 hours & 6 hours \\
\hline \multirow{4}{*}{ An. culicifacies (3) } & Tre- 2.5 & 80 & 75 & 88 & 90 & 95 \\
\hline & Tre- 5 & 88 & 90 & 90 & 96 & 99.2 \\
\hline & Tre- 10 & 97 & 96 & 97 & 100 & 100 \\
\hline & Deet 2.5 & 98 & 100 & 100 & 99 & 100 \\
\hline \multirow{4}{*}{ An. stephensi (3) } & Tre- 2.5 & 84 & 80 & 85.3 & 90 & 96 \\
\hline & Tre- 5 & 89 & 88.7 & 92.0 & 95 & 99 \\
\hline & Tre- 10 & 99.6 & 93.0 & 93.0 & 99 & 100 \\
\hline & Deet 2.5 & 96.7 & 100 & 90 & 99 & 100 \\
\hline \multirow{4}{*}{ Cx. Quinquefasciatus (3) } & Tre- 2.5 & 83.5 & 75 & 82.3 & 91.7 & 95.8 \\
\hline & Tre- 5 & 89.2 & 89.7 & 93 & 95 & 99 \\
\hline & Tre- 10 & 89.6 & 96 & 95.5 & 99 & 100 \\
\hline & Deet 2.5 & 96.6 & 100 & 100 & 96.7 & 100 \\
\hline
\end{tabular}

R- no of Replicate

$\mathrm{N}$ - no of mosquito

other mosquito vector species. The further studies should be investigated against as many different malaria vector as possible under both laboratory and field conditions. Several methods enhancing the efficacy of repellent, such as purification of the active fraction, increase in persistence and duration of repellency need to be studied.

From the observed data on the repellency against the three disease vectors, it can be concluded that dose of $10 \%$ could be used for achieving the desired level of protection against bites of these mosquitoes. However, these results pertain to the effectiveness in cage experiments using only sugar solution as attractant. Thus, further confirmation by testing this repellent on human subjects to evaluate the repellency effect is needed. Our repellent research is being continued to search for the development of new repellents from a natural original that not only offer effective antimosquito products but are also biorational alternatives to synthetic pesticides.

\section{Acknowledgments}

Authors acknowledge the technical help rendered by R. S. Sharma, K. B. Masiwal, and Narender Kumar for the completion of the work. We also thank to Dr. T. G. Thomas, National Institute of Communicable Diseases, New Delhi, India for the critical comments in designing the experiment.

\section{References}

[1] V. P. Sharma, "Re-emergence of malaria in India," Indian Journal of Medical Research, vol. 103, pp. 26-45, 1996.

[2] S. K. Subbarao, N. Nanda, and K. Raghavendra, "Malariogenic stratification of India using Anopheles culicifacies sibling species prevalence," ICMR Bulletin, vol. 29, no. 7, pp. 75-80, 1999.

[3] WHO, International Travel and Health Vaccination Requirement and Health Advice, World Health Organization, Geneva, Switzerland, 1995.
[4] R. K. Gupta and L. C. Rutledge, "Role of repellents in vector control and disease prevention," American Journal of Tropical Medicine and Hygiene, vol. 50, no. 6, supplement, pp. 82-86, 1994.

[5] R. S. Copeland, T. W. Walker, L. L. Robert, J. I. Githure, R. A. Wirtz, and T. A. Klein, "Response of wild Anopheles funestus to repellent-protected volunteers is unaffected by malaria infection of the vector," Journal of the American Mosquito Control Association, vol. 11, no. 4, pp. 438-440, 1995.

[6] E. T. McCabe, W. F. Barthel, S. I. Gertler, and S. A. Halls, "Insect repellents, III. N, N-diethylamides," The Journal of Organic Chemistry, vol. 19, pp. 493-498, 1954.

[7] Anonymous, "Are insect repellents safe?" The Lancet, vol. 2, pp. 610-611, 1988.

[8] M. B. Abou-Donia, K. R. Wilmarth, K. F. Jensen, F. W. Oehme, and T. L. Kut, "Neurotoxicity resulting from coexposure to pyridostimine bromide, DEET, and Permethrin-implications of Golf War chemical exposures," Journal of Toxicology and Environmental Health, vol. 48, pp. 35-36, 1996.

[9] R. W. Haley and T. L. Kurt, "Self-reported exposure to neurotoxic chemical combinations in the Gulf War: a crosssectional epidemiologic study," Journal of the American Medical Association, vol. 277, no. 3, pp. 231-237, 1997.

[10] L. L. Robert, et al., "Comparative sensitivity of four anopheles (Diptera: Culicidae) to five repellents," J. Med. Entomol., vol. 28, no. 3, pp. 417-420, 1991.

[11] M. Sasa, Human Filariasis: A Global Survey of Epidemiology and Control, vol. 819, University of Tokyo Press, Tokyo, Japan, 1976.

[12] V. K. Dua, N. C. Gupta, A. C. Pandey, and V. P. Sharma, "Repellency of Lantana camara (Verbenaceae) flowers against Aedes mosquitoes," Journal of the American Mosquito Control Association, vol. 12, pp. 406-408, 1996.

[13] T. W. Walker, L. L. Robert, R. A. Copeland, et al., "Field evaluation of arthropod repellents, deet and a piperidine compound, AI3-37220, against Anopheles funestus and Anopheles arabiensis in western Kenya," Journal of the American Mosquito Control Association, vol. 12, no. 2, part 1, pp. 172-176, 1996.

[14] R. E. Coleman, L. L. Robert, L. W. Roberts, et al., "Laboratory evaluation of repellents against four anopheline mosquitoes (Diptera: Culicidae) and two phlebotomine sand 
flies (Diptera: Psychodidae)," Journal of Medical Entomology, vol. 30, no. 3, pp. 499-502, 1993.

[15] S. P. Frances, R. D. Cooper, and A. W. Sweeney, "Laboratory and field evaluation of the repellents DEET, CIC-4, and AI3-37220 against Anopheles farauti (Diptera: Culicidae) in Australia," Journal of Medical Entomology, vol. 35, pp. 690-694, 1998.

[16] P. Granett, "Studies of mosquito repellents II Relative performance of certain chemicals and commercially available mixtures as mosquito repellent," Journal of Economic Entomology, vol. 33, pp. 566-572, 1940.

[17] W. Thorsell, A. Mikiver, I. Malander, and H. Tunón, "Efficacy of plant extracts and oils as mosquito repellents," Phytomedicine, vol. 5, no. 4, pp. 311-323, 1998.

[18] L. C. Rutledge, D. M. Collister, V. E. Meixsell, and G. H. C. Eisenberg, "Comparative sensitivity of representative mosquitoes (Diptera: Culicidae) to repellents," Journal of Medical Entomology, vol. 20, pp. 506-510, 1983.

[19] M. A. Ansari and R. K. Razdan, "Relative efficacy of various oils in repelling mosquitoes," Indian Journal of Malariology, vol. 32, no. 3, pp. 104-111, 1995.

[20] D. A. Collins, J. N. Brady, and C. F. Curtis, "Assessment of the efficacy of Quwenling as a mosquito repellent," Phytotherapy Research, vol. 7, no. 1, pp. 17-20, 1993.

[21] V. P. Sharma, M. A. Ansari, and R. K. Razdan, "Mosquito repellent action of neem (Azadirachta indica) oil," American Mosquito Control Association Journal, vol. 9, pp. 359-360, 1993.

[22] B. M. Matsuda, G. A. Surgeoner, J. D. Heal, A. O. Tucker, and M. J. Maciarello, "Essential oil analysis and field evaluation of the citrosa plant "Pelargonium citrosum" as a repellent against populations of Aedes mosquitoes," Journal of the American Mosquito Control Association, vol. 12, no. 1, pp. 69-74, 1996.

[23] M. A. Ansari, P. Vasudevan, M. Tandon, and R. K. Razdan, "Larvicidal and mosquito repellent action of peppermint (Mentha piperita) oil," Bioresource Technology, vol. 71, no. 3, pp. 267-271, 2000.

[24] R. N. Chopra, S. L. Nayar, and I. C. Chopra, Glossary of Indian Medicinal Plants, Publications \& Information Directorate, New Delhi, India, 1956.

[25] N Singh, V. K. Kulshrestha, M. B. Gupta, and K. P. Bhargava, "A pharmacological study of Cyperus rotundus," Indian Journal of Medical Research, vol. 58, pp. 103-109, 1970.

[26] V. P. Sharma and M. A. Ansari, "Personal protection from Mosquitoes (Diptera:Culicidae) by burning neem oil in kerosene repellent action of neem," Journal of Medical Entomology, vol. 31, no. 3, pp. 95-102, 1994.

[27] C. E. Schreck and T. P. Mc Govern, "Repellent tests in the field and laboratory against wild populationsof Mansonia titillans (Diptera: Culicidae)," Journal of Medical Entomology, vol. 22, pp. 658-662, 1985.

[28] H. K. Gouck and C. N. Smith, "The effect of age and time of day on the avidity of Aedes aegypti," The Florida Entomologist, vol. 45, pp. 93-94, 1962.

[29] A. A. Khan, H. I. Maibach, and D. L. Skidmore, "Insect repellents: effect of mosquito and repellent-related factors on protection time," Journal of Economic Entomology, vol. 68, no. 1, pp. 43-45, 1975.

[30] R. D. Xue, D. R. Barnard, and C. E. Schreck, "Influence of body size and age of Aedes albopictuson human host attack rates and the repellency of DEET," Journal of the American Mosquito Control Association, vol. 11, pp. 50-53, 1995.

[31] A. Tawatsin, S. D. Wratten, R. R. Scott, U. Thavara, and Y. Techadamrongsin, "Repellency of volatile oils from plants against three mosquito vectors," Journal of Vector Ecology, vol. 26, no. 1, pp. 76-82, 2001.

[32] S. K. Sharma, V. K. Dua, and V. P. Sharma, "Field study on the mosquito repellent action of neem oil," The Southeast Asian Journal of Tropical Medicine and Public Health, vol. 26, pp. 180-182, 1995.

[33] L. C. Rutledge, M. A. Moussa, C. A. Lowe, and R. K. Sofield, "Comparative sensitivity of mosquito species and strains to the repellent diethyl methybenzamide," Journal of Medical Entomology, vol. 14, pp. 536-541, 1978.

[34] R. A. Bram, "The Genus Culex in Thailand (Diptera: Culicidae)," Contributions of the American Entomological Institute, vol. 12, p. 272, 1967.

[35] K. Tanaka, K. Mizusawa, and E. Saugstad, "Mosquitoes of Japan and Korea," Contributions of the American Entomological Institute, vol. 16, p. 987, 1979.

[36] P. Guptavanij, C. Harinasuta, S. Vutikes, and T. Deesin, "The vectors of periodic and subperiodic Brugia malayi in Thailand Southeast Asian," Journal of Tropical Medicine and Public Health, vol. 2, pp. 589-590, 1971. 

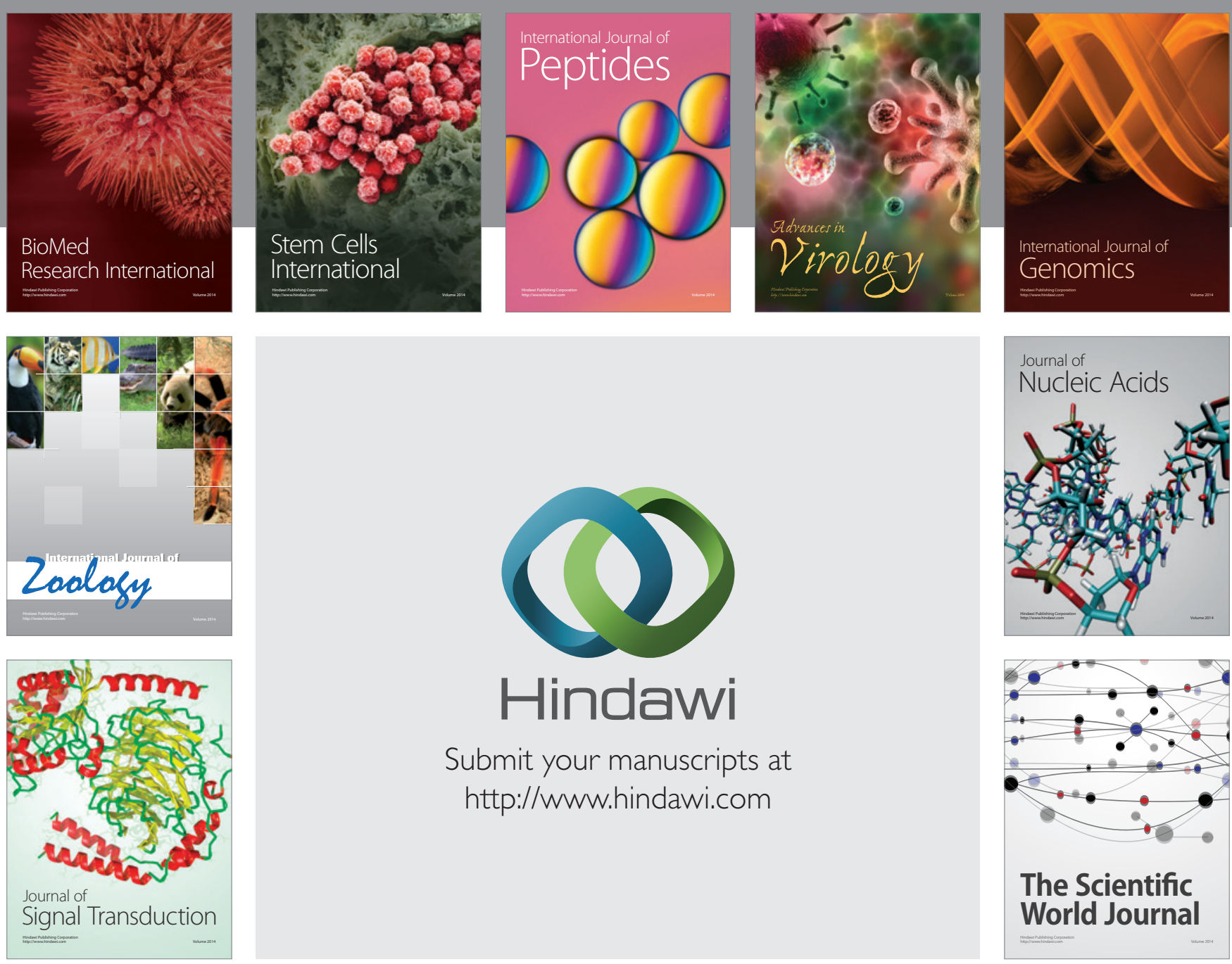

Submit your manuscripts at

http://www.hindawi.com
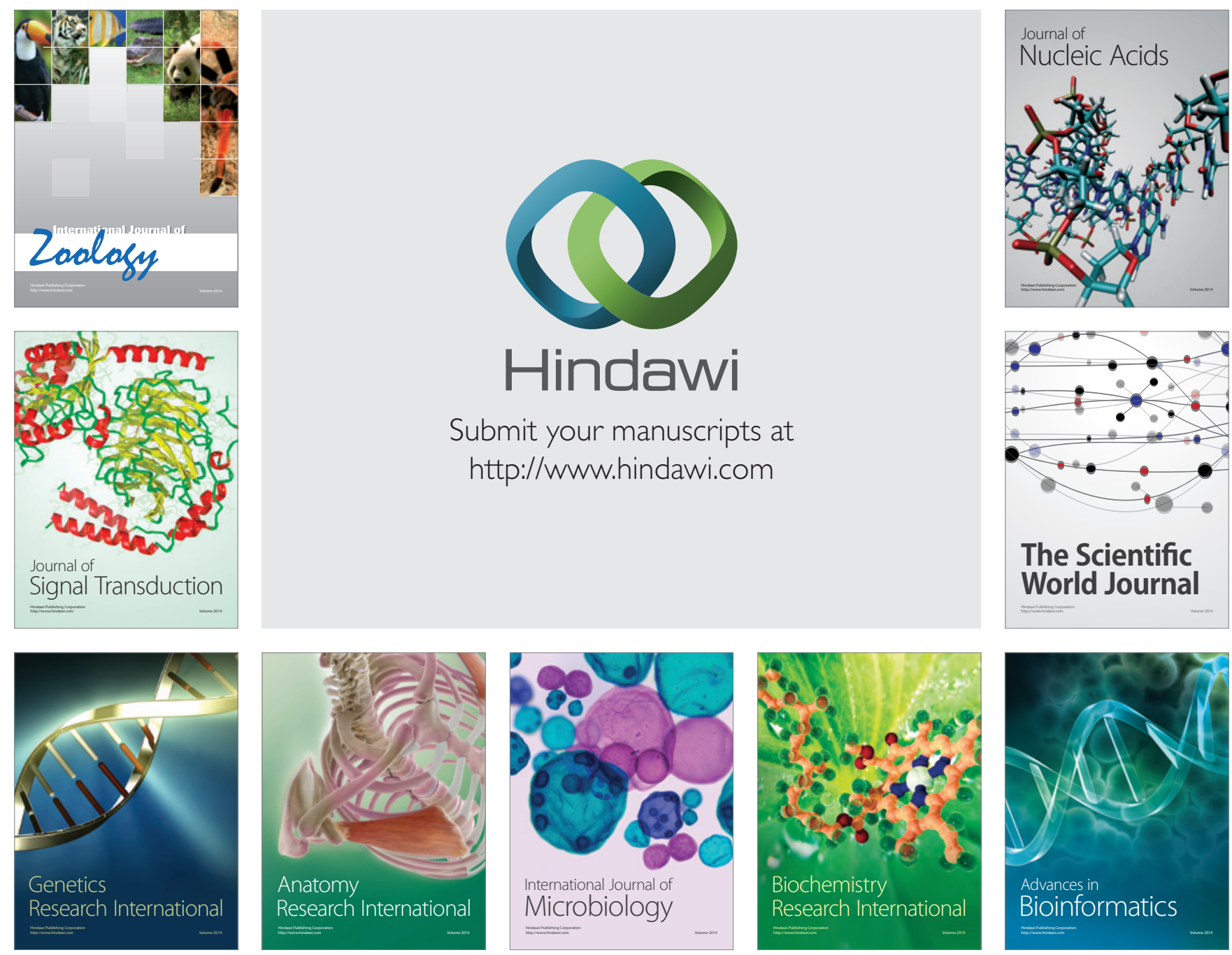

The Scientific World Journal
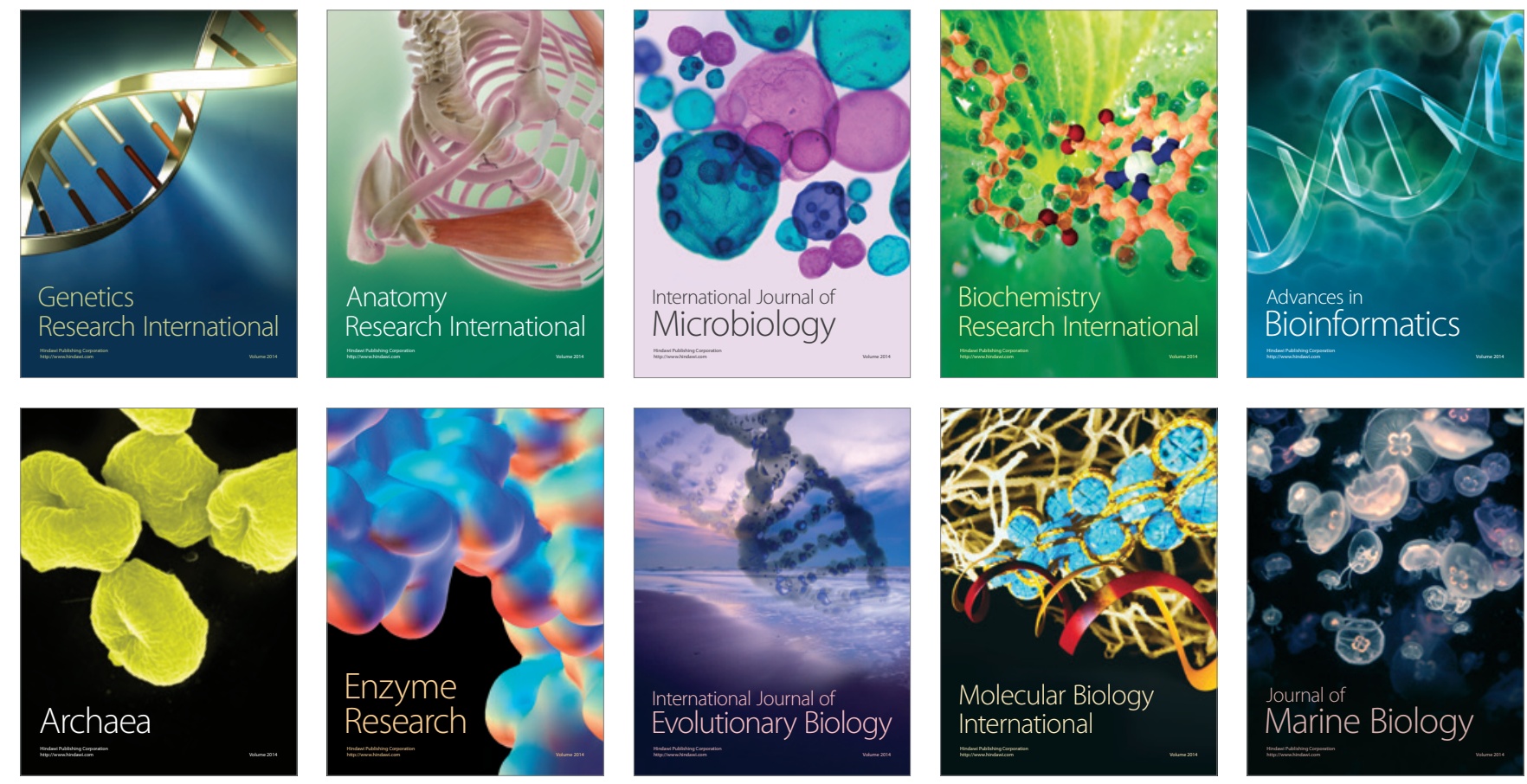